\title{
DOI: http://dx.doi.org/10.21123/bsj.2016.13.1.0082 \\ Synthesis and Characterization of Some New Benzimidazole Derivatives
}

\begin{abstract}
Abdel-Amir M. Fenjan
Sahar T.Adday

Department of Chemistry, College of Science for Women, University of Baghdad $\backslash$ Aljadiyriah $\backslash$ Baghdad $\backslash$ Iraq

E-mail:amirfenjan@Yahoo.com

Received 26, November, 2014

Accepted 14, January, 2015

(c) (7) $\Theta$ This work is licensed under a Creative Commons Attribution-NonCommercialNoDerivatives 4.0 International Licens

Abstract:

In this paper the new starting material 2-(5-chloro- $1 H$-benzo[ $d]$ imidazole-2-yl) aniline (1) was synthesized by the condensation reaction of 4-chloro-o-phenylenediamine and anthranilic acid. The new Mannich base derivatives were synthesized using formaldehyde and different secondary amines to synthesize a new set of benzimidazole derivatives(2-5). Also, the new Schiff-base derivatives (6-10) were synthesized from the reaction of compound (1) with various aromatic aldehydes and the closure-ring was done successfully using mercapto acetic acid to get the new thiazolidine derivatives(11-12).These new compounds were characterized using some physical techniques like:FT-IR Spectra and ${ }^{1}$ HNMR Spectra.
\end{abstract}

Key words: Benzimidazole, Mannich bases, Schiff's bases, Thiazolidine.

\section{Introduction:}

Benzimidazole is a heterocyclic aromatic organic compound. It is a Bicyclic compound composed of the fusion of benzene and imidazole. The most noticeable benzimidazole compound in nature is N-ribosyldimethyl benzimidazole, which used as an axial ligand for cobalt in vitamin $\mathrm{B}_{12}$ [1]. Benzimidazole derivatives have important functions in medical field with many pharmacological activities such as antimicrobial, antiviral, antidiabetic and anticancer activity.It is an important pharmacophore in drug field due to being a good bio activity of naturally forming nucleotides [2-5]. Mannich reactions have become important tools for the synthesis of new compounds. Mannich bases can either be directly used as intermediate chemical synthesis [6].They are organic compounds with the general formula $\mathrm{R}-\mathrm{CH}_{2}-\mathrm{N}<[7][8]$. Also,Schiff's bases are condensation products of primary amines with carbonyl compounds and they were first known by Schiff in 1864 [9].A broad variety of benzimidazole derivatives have been well-known for their chemotherapeutic importance. for example: the presence of mannich side chains in a drug may fight the water bacteria through the formation of hydrochlorides[10] ,schiff's bases have anti cancer activity in animal inspection[11]. 


\section{Materials and Methods:}

Melting points were recorded with Sturat Melting point apparatus and were un corrected.Infra red spectra (FT-IR) were recorded on Shimadzu FT-IR-8300 spectrophotometer in Ibn Sina State Company(ISSC). ${ }^{1} \mathrm{H}-\mathrm{NMR}$ spectra were carried out in Al-al Bayt University (Jordan) operating at 300 $\mathrm{MHz}$ in (DMSO $-\mathrm{d}_{6}$ ) on Fourier transform Varian spectrometer.

\section{Synthesis of Compounds:}

1) Synthesis of 2-(5-chloro- $1 \mathrm{H}$ benzo $[d]$ imidazol-2-yl) aniline (1)

A mixture of a compound 4chloro-1,2-diamino benzene $(0.15 \mathrm{~mol})$ and anthranilic $\operatorname{acid}(0.1 \mathrm{~mol}) w a s$ refluxed with hydrochloric acid $(4 \mathrm{M}$, $80 \mathrm{ml}$ ) for $24 \mathrm{hr}$. The reaction mixture was cooled at the room temperature and neutralized with sodium hydroxide
(10 \%) up to $\mathrm{pH} \mathrm{8-9.} \mathrm{The} \mathrm{obtained}$ solid was filtered off. The Products were purified by recrystallizing from methanol to get beige powder of compound(1), (yield 76\% , M.P (272274) ${ }^{\circ} \mathrm{C}$.

2) Synthesis of Mannich Bases (2-5)

Compound (1) , (2.435 g,0.01 mol) was dissolved in hot methanol (25ml) and the compounds having secondary amine such as indole, isatine , 4-chloro acetanilide, benzanilide were added slowly to this hot solution, then formaldehyde (0.01 mol) was added all at once and refluxed for (4 hours) .The contents were kept overnight in the freezer. The corresponding crystals of mannich bases obtained were recrystallized from ethanol.

Table (1): The physical properties of Mannich bases (2-5)

\begin{tabular}{|c|c|c|c|c|c|}
\hline $\begin{array}{c}\text { Compd. } \\
\text { No. }\end{array}$ & $\begin{array}{c}\text { Nomenclature } \\
\text { and } \\
\text { Chemical formula }\end{array}$ & Structure formule & $\begin{array}{c}\text { Yield } \\
\%\end{array}$ & Color & $\underset{{ }^{\circ} \mathbf{C}}{\mathbf{M} . \mathbf{P}}$ \\
\hline 2 & $\begin{array}{c}\text { [N-((1H-indol-1- } \\
\text { yl)methyl)-2-(5-chloro- } \\
1 H \text {-benzo }[d] \text { imidazole- } \\
2 \text {-yl) aniline }] \\
{\left[\mathrm{C}_{23} \mathrm{H}_{19} \mathrm{ClN}_{4}\right]}\end{array}$ & & 81 & Beige & $122-124$ \\
\hline 3 & $\begin{array}{c}{[1-((2-5 \text {-chloro-1H- }} \\
\text { benzo }[d] \text { imidazole-2- } \\
\text { yl)phenyl amino) } \\
\text { methyl)indoline-2,3- } \\
\text { dione }] \\
{\left[\mathrm{C}_{22} \mathrm{H}_{15} \mathrm{ClN}_{4} \mathrm{O}_{2}\right]} \\
\end{array}$ & & 86 & Red & 118-120 \\
\hline 4 & $\begin{array}{c}\mathrm{N}-((2-5 \text {-chloro-1H- } \\
\text { benzo[d] imidazole-2- } \\
\text { yl)phenyl amino) } \\
\text { methyl)- } \mathrm{N} \text {-(4-chloro } \\
\text { phenyl) acetamide] } \\
{\left[\mathrm{C}_{22} \mathrm{H}_{18} \mathrm{Cl}_{2} \mathrm{~N}_{4} \mathrm{O}\right]}\end{array}$ & & 70 & brown & 130-132 \\
\hline 5 & $\begin{array}{c}\text { [N-((2-5-chloro-1H- } \\
\text { benzo[d] imidazole-2- } \\
\text { yl)phenyl amino) } \\
\text { methyl)-N-phenyl } \\
\text { benzamide] } \\
{\left[\mathrm{C}_{27} \mathrm{H}_{21} \mathrm{ClN}_{4} \mathrm{O}\right]}\end{array}$ & & 55 & $\begin{array}{l}\text { Light } \\
\text { brown }\end{array}$ & 138-140 \\
\hline
\end{tabular}

\section{3) Synthesis of Schiff Bases (6-10)}

A mixture of compound (1) $(0.2435 \mathrm{~g}$ $, 0.001 \mathrm{~mol})$ and aldehydes $(0.001 \mathrm{~mol})$ in absolute ethanol $(15 \mathrm{ml})$ and (3)drops of glacial acetic acid were refluxed for (6 hours). The mixture was cooled, collected by filtration and recrystallized from methanol. 
Table (2): The physical properties of Schiff bases (6-10)

\begin{tabular}{|c|c|c|c|c|c|}
\hline $\begin{array}{c}\text { Compd. } \\
\text { No. }\end{array}$ & \begin{tabular}{|c|} 
Nomenclature \\
and \\
Chemical formula \\
\end{tabular} & Compound Structure & $\begin{array}{c}\text { Yield } \\
\%\end{array}$ & Color & $\underset{{ }^{\circ} \mathrm{C}}{\mathrm{M} . \mathrm{P}}$ \\
\hline 6 & $\begin{array}{c}\text { [N-benzilidine-(2-(5-chloro- } \\
1 H \text {-benzo }[d] \text { imidazol-2- } \\
\text { yl)aniline }] \\
{\left[\mathrm{C}_{20} \mathrm{H}_{14} \mathrm{ClN}_{3}\right]} \\
\end{array}$ & & 62 & $\begin{array}{l}\text { Light } \\
\text { yellow }\end{array}$ & $160-162$ \\
\hline 7 & $\begin{array}{c}\text { [4-((2-(5-chloro-1H- } \\
\text { benzo[d]imidazol } \\
\text {-2-yl)phenyl imino) methyl } \\
\text { phenol] } \\
{\left[\mathrm{C}_{20} \mathrm{H}_{14} \mathrm{ClN}_{3} \mathrm{O}\right]}\end{array}$ & & 73 & Beige & 228-230 \\
\hline 8 & $\begin{array}{c}\text { [N-(4-bromo benzilidine)- 2- } \\
\text { (5-chloro-1H-benzo }[d] \\
\text { imidazol-2-yl)aniline }] \\
{\left[\mathrm{C}_{20} \mathrm{H}_{13} \mathrm{BrClN}_{3}\right]}\end{array}$ & & 79 & $\begin{array}{c}\text { Dark } \\
\text { yellow }\end{array}$ & 212-214 \\
\hline 9 & $\begin{array}{c}\text { [2-(5-chloro- } 1 \mathrm{H} \text { - } \\
\text { benzo[d]imidazol } \\
-2 \text {-yl) - } \mathrm{N} \text {-(4-chloro } \\
\text { benzilidine)aniline }] \\
{\left[\mathrm{C}_{20} \mathrm{H}_{13} \mathrm{Cl}_{2} \mathrm{~N}_{3}\right]}\end{array}$ & & 83 & $\begin{array}{l}\text { Light } \\
\text { yellow }\end{array}$ & 206-208 \\
\hline 10 & $\begin{array}{c}\text { [4-(2-(5-chloro-1H- } \\
\text { benzo[ }[d] \text { imidazol } \\
\text {-2-yl) phenyl imino)methyl)- } \\
\text { N,N-dimethylaniline] } \\
{\left[\mathrm{C}_{22} \mathrm{H}_{19} \mathrm{ClN}_{4}\right]} \\
\end{array}$ & & 81 & Yellow & 218-220 \\
\hline
\end{tabular}

4) Synthesis of Compounds [(2-Aryl4-Oxo-1,3-Thiazolidin)- Hydrazino Acetyl] benzimidazole (11-12)

A mixture of the compounds (9or10) (0.01 mmole), $10 \mathrm{ml}$ absolute ethanol were refluxed for 15 minutes. Then mercapto acetic acid ( 0.01 mmole) was added drop wise during 15 minutes while the mixture is hot. After that the mixture was refluxed for $10 \mathrm{hr}$, evaporated and treated with saturated solution of sodium bicarbonate filtered off and set aside at R.T up to dryness and recrystallized from ethanol to give the thiazolidine compounds.

Table (3): The physical properties of compound (11-12)

\begin{tabular}{|c|c|c|c|c|c|}
\hline $\begin{array}{l}\text { Compd. } \\
\text { No. }\end{array}$ & Nomenclature & Structure formula & $\begin{array}{l}\text { Yield } \\
\%\end{array}$ & Color & $\begin{array}{l}\text { M. P. } \\
{ }^{\circ} \mathrm{C}\end{array}$ \\
\hline 11 & $\begin{array}{l}\text { [3-(2-(5-chloro-1H- } \\
\text { benzo[d]imidazol } \\
\text {-2-yl)phenyl)-2- } \\
\text { (4-chloro phenyl) } \\
\text { thiazolidin-4-one] } \\
{\left[\mathrm{C}_{22} \mathrm{H}_{15} \mathrm{Cl}_{2} \mathrm{~N}_{3} \mathrm{OS}\right]}\end{array}$ & & 94 & Yellow & 232-234 \\
\hline 12 & $\begin{array}{l}{[3-(2-(5 \text {-chloro- } 1 \mathrm{H}-} \\
\text { benzo[d]imidazol } \\
-2-y l) \text { phenyl)-2- } \\
(4-d i \text { methyl amino }) \\
\text { phenyl) } \\
\text { thiazolidin -4-one }] \\
{\left[\mathrm{C}_{24} \mathrm{H}_{21} \mathrm{ClN}_{4} \mathrm{OS}\right]}\end{array}$ & & 92 & Orange & 249-251 \\
\hline
\end{tabular}

Spectroscopic data of the synthesized compounds:

The structure of the synthesized compounds has been characterized and confirmed by FT-IR spectra, and ${ }^{1}$ H.NMR spectra for some of them.
2-(5-chloro-1H-benzo $[d]$ imidazol-2yl) aniline (1) FTIR (KBr) cm ${ }^{-1}$ : 34063433 $\left(\mathrm{NH}_{2}\right), 3329(\mathrm{NH}), 3070(\mathrm{ArC}-\mathrm{H})$, $1608 \quad(-\mathrm{C}=\mathrm{N}), \quad 752(\mathrm{C}-\mathrm{Cl}) .{ }^{1} \mathrm{HNMR}$

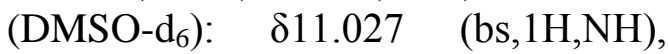


$\delta(6.1-6.9)(\mathrm{m}, 7 \mathrm{H}, \mathrm{C}-\mathrm{H}$ aromatic $), \delta$ $4.043\left(\mathrm{~s}, 1 \mathrm{H}, \mathrm{NH}_{2}\right)$.

N-((1H-indol-1-yl)methyl)-2-(5chloro- $1 H$-benzo $[d]$ imidazole-2yl)aniline(2)

FTIR(KBr) $\mathrm{cm}^{-1}: \quad 3417$ (NH)Amine, (3402,3290) (NH) Imidazole(taut.), 3082 (Ar-C-H), 2962 (Aliphatic-C-H), $1612(-\mathrm{C}=\mathrm{N}), 744(\mathrm{C}-\mathrm{Cl})$.

1-((2-5-chloro-1H-benzo[d]

imidazole-2-yl) phenyl amino) methyl) indoline-2,3-dione (3)

FTIR (KBr) $\mathrm{cm}^{-1}$ : 3394 (NH)Amine ,3244 (NH)Imidazole, 3066(Ar-C-H) , 2947 (Aliphatic-C-H), $1612(-\mathrm{C}=\mathrm{N})$, $1735(\mathrm{C}=0), \quad 752 \quad(\mathrm{C}-\mathrm{Cl}) .{ }^{1} \mathrm{HNMR}$ $\left(\mathrm{DMSO}^{\left.-\mathrm{d}_{6}\right):} \delta 4.8 \quad\left(\mathrm{~s}, 2 \mathrm{H}, \mathrm{CH}_{2}-\mathrm{N}\right), \quad \delta\right.$ $(6.2-8.2)(\mathrm{m}, 11 \mathrm{H}$, aromatic $\mathrm{C}-\mathrm{H}), \delta 3.7$ $\left(\mathrm{s}, 1 \mathrm{H}, \mathrm{NH}-\mathrm{CH}_{2}\right), \delta 10.75(\mathrm{~s}, 1 \mathrm{H}, \mathrm{NH}$ of imidazole).

N-((2-5-chloro-1H-

benzo $[d]$ imidazole-2-

yl)phenylamino)methyl)-N-(4-

chlorophenyl)acet amide (4) FTIR $(\mathrm{KBr}) \mathrm{cm}^{-1}$ : 3302 (NH)Amine ,3263, 3194 (NH)Imidazole (taut.), 3078(ArC-H) , 2981 (Aliphatic-C-H), 1604 ($\mathrm{C}=\mathrm{N}), 1666(\mathrm{C}=\mathrm{O}), 752(\mathrm{C}-\mathrm{Cl}) .{ }^{1} \mathrm{HNMR}$ $\left(\mathrm{DMSO}_{-} \mathrm{d}_{6}\right): \delta(3.8-4.2)\left(\mathrm{s}, 2 \mathrm{H}, \mathrm{CH}_{2^{-}}\right.$ $\mathrm{N}), \delta(6.9-8.9)(\mathrm{m}, 11 \mathrm{H}$, aromatic $\mathrm{C}-\mathrm{H})$, $\delta 4.9\left(\mathrm{~s}, 1 \mathrm{H}, \mathrm{NH}-\mathrm{CH}_{2}\right), \delta 10.75(\mathrm{~s}, 1 \mathrm{H}$, $\mathrm{NH}$ of imidazole).

N-((2-5-chloro-1H-benzo[d]

imidazole-2-yl) phenyl amino) methyl) -N-phenyl benzamide (5)

FTIR (KBr) cm $\mathrm{cm}^{-1}$ 3475(NH)Amine, 3344, 3124 (NH) Imidazole(taut.), 3051 (Ar-C-H), 2789 (Aliphatic-C-H), $1654(\mathrm{C}=\mathrm{O}), 1600(-\mathrm{C}=\mathrm{N}), 752(\mathrm{C}-\mathrm{Cl})$. ${ }^{1} \mathrm{HNMR}\left(\mathrm{DMSO}-\mathrm{d}_{6}\right): \quad \delta(3.9-4.16)(\mathrm{s}$, $\left.2 \mathrm{H}, \mathrm{CH}_{2^{-}} \mathrm{N}\right), \delta(6.9-8.9)(\mathrm{m}, 11 \mathrm{H}$, aromatic $\mathrm{C}-\mathrm{H}), \delta 4.9\left(\mathrm{~s}, 1 \mathrm{H}, \mathrm{NH}-\mathrm{CH}_{2}\right), \delta$ 10.75 (s,1H,NH of imidazole).

N-benzilidine-(2-(5-chloro-1H-

benzo $[d]$ imidazol-2-yl) aniline (6) FTIR (KBr) $\mathrm{cm}^{-1}: \quad 3367,3194 \quad(\mathrm{NH})$ Imidazole (taut.), 3082 (Ar-C-H), 2935 (Aliphatic-C-H), 1658, $1612(-\mathrm{C}=\mathrm{N})$, 752 (C-Cl) .
4-((2-(5-chloro-1H-benzo[d]imidazol2-yl) phenylimino) methylphenyl (7) FTIR(KBr) $\mathrm{cm}^{-1}: 3421$ (C-O-H) , 3383, 3325 (NH)Imidazole (taut.), 3074 (ArC-H), 2835 (Aliphatic-C-H), 1678, $1608(-\mathrm{C}=\mathrm{N}), 750(\mathrm{C}-\mathrm{Cl})$.

N-(4-bromo benzilidine)- 2-(5chloro-1H -benzo[d]imidazol-2yl)aniline (8) FTIR (KBr) $\mathrm{cm}^{-1}: 3360$, 3329 (NH) Imidazole (taut.), 3078(ArC-H), 2850 (Aliphatic-C-H), 1608, $1573(-\mathrm{C}=\mathrm{N}), 756(\mathrm{C}-\mathrm{Cl}), 524(\mathrm{C}-\mathrm{Br})$.

[2-(5-chloro-1H-benzo[d]imidazol-2-

yl) -N-(4-chloro benzilidine)aniline

(9) FTIR $(\mathrm{KBr}) \mathrm{cm}^{-1}: 3452,3402(\mathrm{NH})$ Imidazole (taut.),3097(Ar-C-H), 2931 (Aliphatic-C-H), 1610(-C=N),748 (C$\mathrm{Cl})$.

[4-(2-(5-chloro-1H-benzo[d]imidazol2-yl)phenylimino)methyl)-N,Ndimethylaniline (10) FTIR $(\mathrm{KBr}) \mathrm{cm}^{-}$ ${ }^{1}: 3078$ (Ar-C-H), 2962 (Aliphatic-C$\mathrm{H}), 1612 \quad(-\mathrm{C}=\mathrm{N}), 748(\mathrm{C}-\mathrm{Cl}) .{ }^{1} \mathrm{HNMR}$ $\left(\mathrm{DMSO}_{6} \mathrm{~d}_{6}\right): \delta 3.4\left(\mathrm{~s}, 6 \mathrm{H}, \mathrm{N}\left(\mathrm{CH}_{3}\right)_{2}\right), \delta$ $8.4(\mathrm{~s}, 1 \mathrm{H}, \mathrm{CH}=\mathrm{N}), \delta(6.15-8)(\mathrm{m}, 11 \mathrm{H}$, aromatic C-H), $\delta 11.027(\mathrm{~s}, 1 \mathrm{H}, \mathrm{NH}$ of imidazole).

3-(2-(5-chloro-1H-benzo[ $d]$ imidazol2-yl)phenyl)-2-(4-chlorophenyl) thiazolidin-4-one(11)

FTIR(KBr) $\quad \mathrm{cm}^{-1}: 3429,3365 \quad(\mathrm{NH})$ Imidazole(taut.), 3078 (Ar-C-H), 2981 (Aliphatic-C-H), $1732(\mathrm{C}=\mathrm{O}), 1577$ ( $-\mathrm{C}=\mathrm{N}), \quad 783,767(\mathrm{C}-\mathrm{Cl}), 524(\mathrm{C}-\mathrm{S})$. ${ }^{1} \mathrm{HNMR}$ (DMSO-d6) : $\delta 3.4$ (s,2H,S$\left.\mathrm{CH}_{2}\right), \delta \quad 6.1 \quad(\mathrm{~s}, 1 \mathrm{H}, \mathrm{N}-\mathrm{CH}), \delta$ (7-7.9) $(\mathrm{m}, 11 \mathrm{H}$, aromaticCH), $\delta 9.2(\mathrm{~s}, 1 \mathrm{H}, \mathrm{OH})$ , $\delta 11.027$ (s,1H,NH of imidazole).

3-(2-(5-chloro-1H-benzo[d]imidazol2-yl) phenyl)-2-(4-di methyl amino) phenyl) thiazolidin -4-one (12)

FTIR (KBr) $\quad \mathrm{cm}^{-1}: 3429,3365 \quad(\mathrm{NH})$ Imidazole (taut.), 3078(Ar-C-H) , 2981 (Aliphatic-C-H), $1732 \quad(\mathrm{C}=\mathrm{O}), 1577$ ($\mathrm{C}=\mathrm{N}), \quad 783,767(\mathrm{C}-\mathrm{Cl}), \quad 524 \quad(\mathrm{C}-\mathrm{S})$. ${ }^{1} \mathrm{HNMR} \quad$ (DMSO-d6): $\quad \delta 3 \quad(\mathrm{~s}, 6 \mathrm{H}, \mathrm{N}$ $\left.\left(\mathrm{CH}_{3}\right)_{2}\right), \delta 3.4\left(\mathrm{~s}, 2 \mathrm{H}, \mathrm{S}-\mathrm{CH}_{2}\right), \delta 4.7(\mathrm{~s}$, $1 \mathrm{H}, \mathrm{N}-\mathrm{CH}), \delta(6.7-7.9)(\mathrm{m}, 11 \mathrm{H}$, aromatic $\mathrm{CH}), \delta 9.2(\mathrm{~s}, 1 \mathrm{H}, \mathrm{OH}), \delta 11.156(\mathrm{~s}, 1 \mathrm{H}, \mathrm{NH}$ of imidazole). 


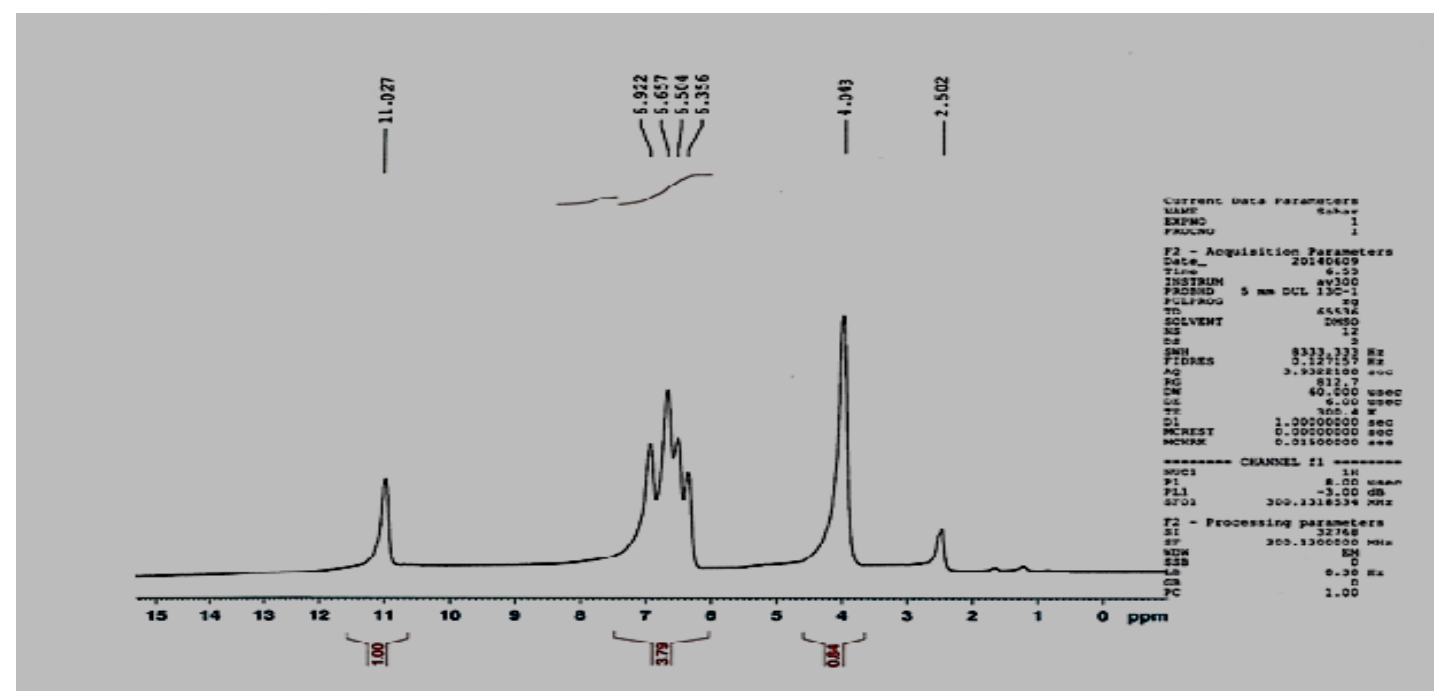

Fig.(1): ${ }^{1}$ H-NMR Spectrum of compound (1)

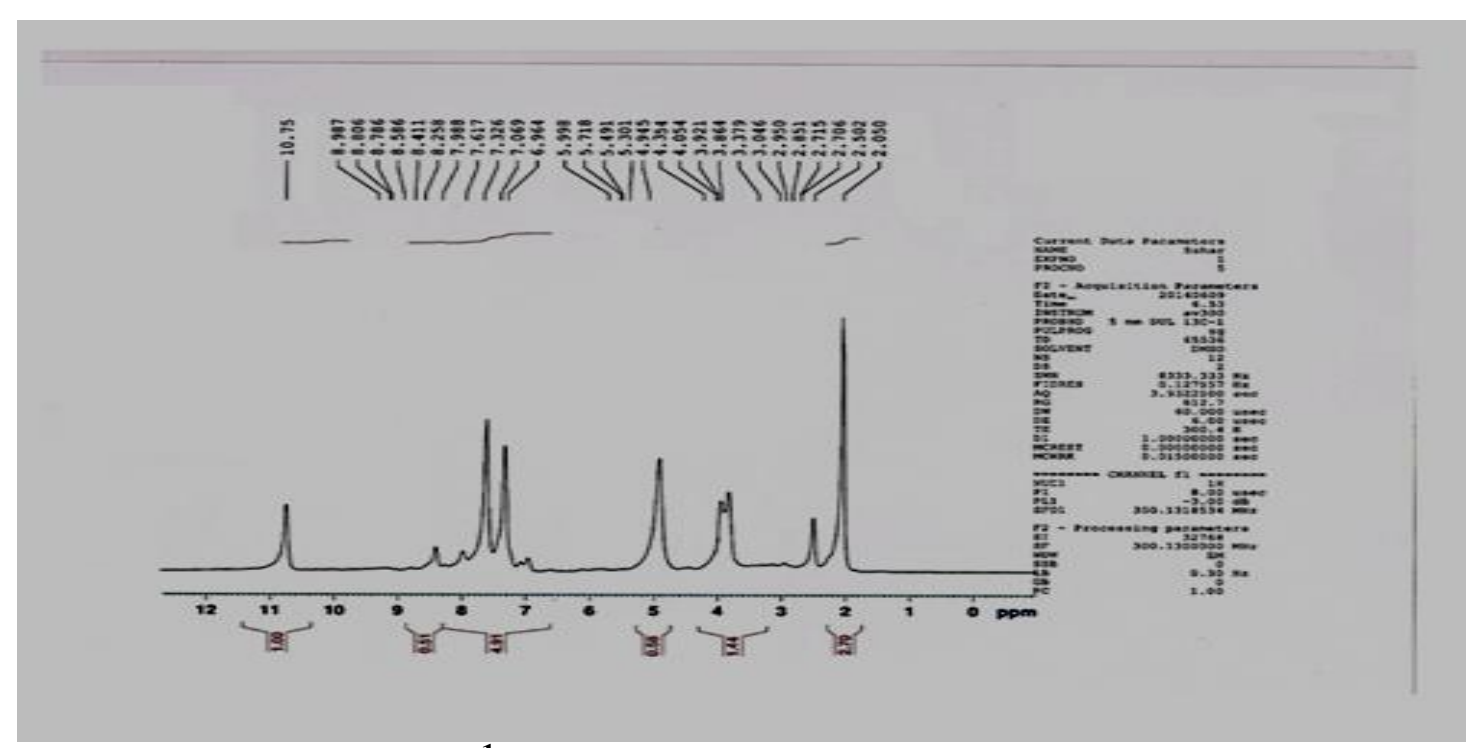

Fig.(2): ${ }^{1}$ H-NMR Spectrum of compound (4)

\section{Results and Discussion:}

This paper reports a simple and an effective method for the synthesis of some new benzimidazole derivatives which were obtained in good to excellent yield. In the present work 2(5-chloro-1 $H$-benzo $[d]$ imidazole-2yl)aniline (1)which was synthesized from the condensation reaction of 4chloro-o-phenylenediamine with anthranilic acid was used as the key intermediate for further synthesis Thus, the compound (1)was allowed to undergo the Mannich reaction with different secondary amines namely indole, isatine, 4-chloro acetanilide, and benzanilide using formaldehyde in absolute methanol to give compounds (2-5) respectively. Also, the condensation reaction of the compound (1) with different aromatic aldehydes ,namely, benzaldehye,4-hydroxybenzaldehyde, 4-bromo benzaldehyde, 4-chloro Benzaldehyde, and $\mathrm{N}$,Ndimethyl benzaldehyde in absolute ethanol afforded the corresponding Schiff's bases(6-10). On the other hand, the cyclo condensation of some substituted Schiff's bases $(9,10)$ with thioglygolic acid afforded the corresponding thiazolidine $(11,12)$ respectively [12] .The reactions sequence was illustrated in the scheme (1) : 


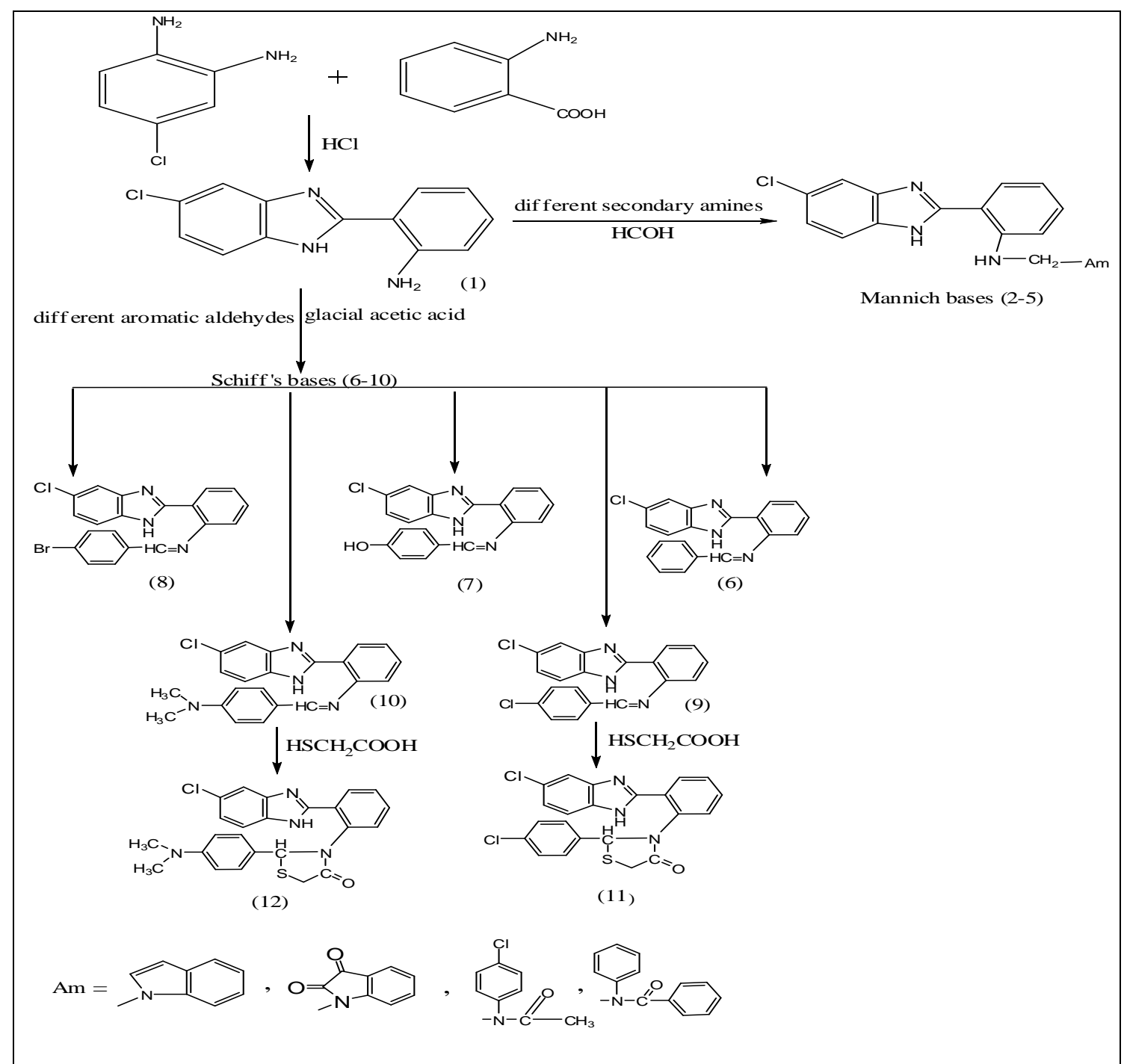

Scheme (1) : The reactions sequence for the synthesis of some new benzimidazole derivatives

\section{References:}

[1] Barker, H. A.; Smyth, R. D., Weissbach, H.; Toohey, J. I.; Ladd, J. N. and Volcani, B. E. 1960. Isolation and Properties of Crystalline Cobamide Coenzymes Containing Benzimidazole or 5,6Dimethylbenzimidazole, J. Biol. Chem. 235 (2): 480-488.

[2] Galal, S.A.; Abdelsamiea, A.S.; Rodriguez, M. L.; Kerwin, S. M. and ElDiwani,H.I.2010. Synthesis and studying the antitumor activity of novel 5- (2-methylbenzimidazol5-yl)-1,3,4- oxadiazole-2(3H)thiones, Eur J Chem .(2): 67-72.
[3] Bhatnagar, A.; Sharma, P. K. and Kumar, N. 2011. A Review on "Imidazoles": Their Chemistry and Pharmacological Potentials, Int.J. Pharm Tech Res. 3 (1):268-282.

[4] Maru, M. and Shah, M. K.2012. Transition metal complexes of 2(substituted-1H-pyrazole-4-yl)-

$1 H$ benzo[ $d]$ imidazoles: Synthesis and characterization, J. Chem. Pharm. Res.4 (3):1638-1643.

[5] Chawla, A.; Sharma, A. and Sharma, A. K. 2012. Review: A convenient approach for the synthesis of imidazole derivatives using microwaves, DPC. 4 (1):116140. 
[6] Vogel,S. and Heilmann, J .2008. Synthesis, cytotoxicity, and antioxidative activity of minor prenylate chalcones from Humulus lupulus, J. Nat. Prod. 71: 12371241.

[7] Babasaheb, P.B.; Sachin, A. P. and Rajesh, N.G. 2010. Synthesis and biological evaluation of nitrogen containing chalcones as possible anti-inflammatory and antioxidant agents, Bioorg. M Chem. Lett. 20: 730-733.

[8] Kim, Y. H.; Kim, J.; Park, H. and Kim, H.P. 2007. Anti-inflammatory activity derivatives: Inhibition of inducible nitric oxide synthasecatalyzed nitric oxide production from lipopoly saccharide-treated RAW 264.7 cells, Biol. Pharm.Bull. 30, 1450-1455.
[9] Cimerman, Z.; Miljanic, S. and Galic, N. 2000. Schiff bases derived from amino pyridines as spectro fluorimetric analytical reagents, CCA. 73(1): 81-95.

[10] Kamel, M. M. and Naser, M. E. 1979. Synthesis and Antimicrobial activity of some new benzimidazole derivatives, Pharmazie. 34(7) : 440.

[11]Popp, F. D. 1961. Synthesis of Potential Anticancer Agents. II. Some Schiff Bases, j.Org.Chem. 26 (1): 1566-1568.

[12] Mickeviciene, K.; Voskiene, A. and Mickevicius,V.2014. Synthesis of some 1- and 2-carboxyalkyl substituted benzimidazoles and their derivatives, Res Chem Intermed. 40:1619-1631.

\section{تحضير وتشخيص بعض المشتقات الجديدة من البنزايميدازول

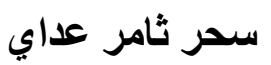 \\ عبد الامير مطلك فنجان \\ قسم الكيمياء /كلية العلوم للبنات / جامعة بغداد/الجادرية / بغداد/ العر اق}

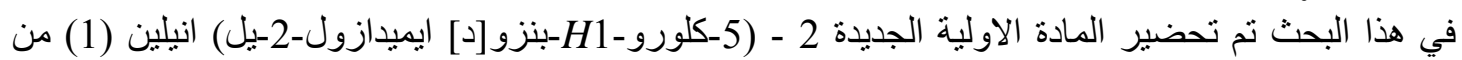

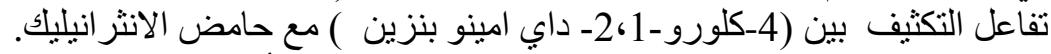

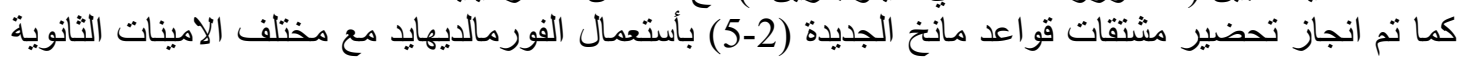
لتحضير مجمو عة جديدة من مشتقات البنزو ايميدازول.

كذللك تم تحضير مشتقات قاعدة شف الجديدة( 6-10) من تفاعل مختلف الالديهايدات مع المادة الاولية الجديدة

و تم غلق المشتقات الجديدة لقو اعد شف (12-10) (12) بنجاح بأستعمال (2- مركبتو حامض الخليك) للحصول على

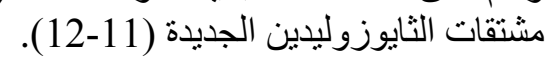

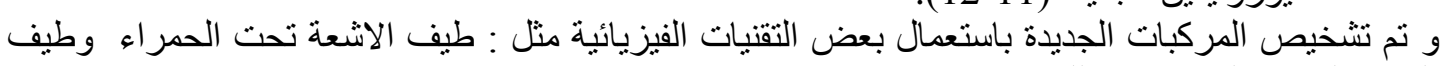

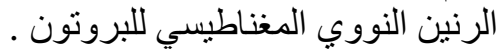

الكلمات المفتاحية : بنز ايميدازول ، قو اعد مانخ ، قو اعد شف ، ثايوزوليدين . 